\title{
Variabilidade espacial da estabilidade de agregados e matéria orgânica em solos de relevos diferentes
}

\author{
Zigomar Menezes de Souza ${ }^{(1)}$, José Marques Júnior ${ }^{(1)}$ e Gener Tadeu Pereira(2)
}

\begin{abstract}
(1)Universidade do Estado de São Paulo (Unesp), Fac. de Ciências Agrárias e Veterinárias, Dep. de Solos e Adubos, Via de acesso Prof. Paulo Donato Castellane, s/no, CEP 14870-900 Jaboticabal, SP. E-mail: zigomar@fcav.unesp.br, marques@fcav.unesp.br (2)Unesp, Fac. de Ciências Agrárias e Veterinárias, Dep. de Ciências Exatas. E-mail: genertp@fcav.unesp.br
\end{abstract}

\begin{abstract}
Resumo - Solos submetidos ao mesmo sistema de manejo em locais com pequena variação de relevo manifestam variabilidade espacial de atributos diferenciada. $O$ objetivo deste trabalho foi avaliar a variabilidade espacial do diâmetro médio geométrico de agregados na classe $>2 \mathrm{~mm}$, na classe $2-1 \mathrm{~mm}$ e da matéria orgânica em um Latossolo Vermelho eutroférrico sob cultivo de cana-de-açúcar. Foram feitas amostragens do solo em intervalos regulares de $10 \mathrm{~m}$, em forma de malha, totalizando 100 pontos, coletadas nas profundidades de $0,0-0,2 \mathrm{me} 0,2-0,4 \mathrm{~m}$. Os dados foram submetidos à análise estatística descritiva, geoestatística e krigagem. Em relação à matéria orgânica, os valores do coeficiente de variação foram baixos na profundidade de $0,0-0,2 \mathrm{~m}$ e médio na profundidade de $0,2-0,4 \mathrm{~m}$; foi alto para o diâmetro médio geométrico e médio para agregados na classe $>2 \mathrm{~mm}$ e agregados na classe $2-1 \mathrm{~mm}$. Observou-se dependência espacial em todas as variáveis, e os maiores alcances foram observados na profundidade de $0,0-0,2 \mathrm{~m}$ nas variáveis estudadas. Pequenas variações no gradiente do declive e nas formas do relevo condicionaram variabilidade espacial diferenciada da matéria orgânica e estabilidade de agregados nas profundidades estudadas.
\end{abstract}

Termos para indexação: Saccharum officinarum, geoestatística, krigagem, relação solo-paisagem, Latossolo.

\section{Spatial variability of the stability of aggregates and organic matter in soils with different relieves}

\begin{abstract}
Soils submitted to the same management system in places with small relief variation manifest different spatial variability on their attributes. The objective of this work was to evaluate the spatial variability of the geometric medium diameter, aggregates in the $>2 \mathrm{~mm}$ class, aggregates in the 2-1 mm class and organic matter of an Oxisol under culture of the sugarcane. Samplings of the soil in regular intervals of $10 \mathrm{~m}$, in grid form, totaling 100 points, collected in the depths of $0.0-0.2 \mathrm{~m}$ and $0.2-0.4 \mathrm{~m}$ were made. Data were submitted to descriptive statistics, geostatistics and in sequence to kriging analyzes. Values of the variation coefficient were low for organic matter in the depth of $0.0-0.2 \mathrm{~m}$, mean in the depth of $0.2-0.4 \mathrm{~m}$, high for geometric medium diameter and mean for aggregates in the $>2 \mathrm{~mm}$ class, aggregates in the $2-1 \mathrm{~mm}$ class in all studied depths. The occurrence of space dependence was observed for all the variables, and the largest ones were observed in the depth of $0.0-0.2 \mathrm{~m}$. Small variations in the forms of the relief condition spatial variability differentiated for organic matter and stability of aggregates in the studied depths.
\end{abstract}

Index terms: Saccharum officinarum, geostatistics, kriging, soil-landscape relationship, oxisols.

\section{Introdução}

A formação e a estabilização dos agregados do solo ocorrem simultaneamente na atuação de processos físicos, químicos e biológicos. Esses processos atuam por mecanismos próprios, em que são envolvidos por substâncias que agem na agregação e na estabilização. Entre essas, as principais são: argila, sílica coloidal, compostos orgânicos, metais polivalentes, carbonato de cál- cio, óxido e hidróxidos de ferro e alumínio (Silva \& Mielniczuk, 1997).

O uso intensivo dos Latossolos Vermelho eutroférricos no nordeste do Estado de São Paulo no cultivo da canade-açúcar, com preparo superficial excessivo e queima dos resíduos, modifica significativamente as propriedades físicas do solo, principalmente em relação à estabilidade de agregados. A manutenção de uma boa es- 
tabilidade de agregados é condição primordial para garantir altas produtividades (Perin et al., 2002).

Uma alternativa do setor sucroalcooleiro na redução de custos é a adoção da agricultura de precisão, que promove conhecimento da variabilidade dos solos. A variabilidade dos solos tem sido abordada pela classificação numérica, por métodos de estatística multivariada, classificação contínua (fuzzy), geoestatística, métodos fractais, morfologia matemática e teoria do caos (Burrough et al., 1994). Embora estes métodos estatísticos permitam inferir sobre a variabilidade espacial do solo (vertical e horizontal), a dependência espacial entre as amostras somente pode ser modelada por meio da geoestatística (Mulla et al., 1992; Webster, 2000). Análises estatísticas clássicas que consideram a independência entre as amostras, baseadas na média, vêm sendo substituídas por análises geoestatísticas fundamentadas na teoria das variáveis regionalizadas (Isaaks \& Srivastava, 1989), por intermédio do semivariograma e da dependência espacial.

O estudo dos atributos físicos e químicos do solo sempre foi importante instrumento no processo de análise de produtividade e da conseqüente escolha sobre técnicas de manejo a serem adotadas em uma determinada área (Pierce \& Nowak, 1999).

O entendimento da variabilidade exige uma maior quantidade de informações, as quais podem ser obtidas a partir de operações de amostragem de solo no campo. Quanto maior o número de amostras, mais alto é o ônus financeiro para o agricultor, e, portanto, o método da geoestatística é uma alternativa na otimização deste processo (Mulla et al., 1992). O conhecimento das relações entre solo e a posição da paisagem definida no espaço e no tempo pode subsidiar levantamentos de solos (Marques Júnior \& Lepsch, 2000). A paisagem pode ser utilizada na definição de zonas de manejo, permitindo definição de práticas regionalizadas do solo, e, juntamente com a definição da variabilidade espacial, possibilita melhor controle dos fatores de produção das culturas e proteção ambiental (Fraisse et al., 1999).

O objetivo deste trabalho foi avaliar a variabilidade espacial do diâmetro médio geométrico, agregado na classe $>2 \mathrm{~mm}$, na classe 2-1 mm e da matéria orgânica em um Latossolo Vermelho eutroférrico, sob cultivo de cana-de-açúcar em áreas com pequenas variações no gradiente do declive e nas formas do relevo.

\section{Material e Métodos}

A área de estudo localiza-se no nordeste do Estado de São Paulo, no Município de Guariba, SP. As coordenadas geográficas são $21^{\circ} 19^{\prime} \mathrm{S}$ e $48^{\circ} 13^{\prime} \mathrm{O}$, com altitude média de $640 \mathrm{~m}$ acima do nível do mar. O clima da região, segundo a classificação de Köppen, é do tipo mesotérmico com inverno seco (Cwa), com precipitação média de $1.400 \mathrm{~mm}$, com chuvas concentradas no período de novembro a fevereiro. A vegetação natural era constituída por floresta tropical subcaducifólia.

O relevo é predominantemente suave ondulado com declividades médias variando de $3 \%$ a $8 \%$. A área experimental está sob cultivo de cana-de-açúcar há mais de trinta anos. O solo da área foi classificado como Latossolo Vermelho eutroférrico, textura muito argilosa (Embrapa, 1999).

De acordo com o modelo de Troeh (1965), classificou-se a curvatura e o perfil das formas do terreno no terço inferior da encosta em dois compartimentos, I e II (Figura 1). No compartimento I, a forma do relevo é basicamente linear e o compartimento II apresenta curvaturas côncavas e convexas. O solo foi amostrado nos pontos de cruzamento de uma malha com intervalos regulares de $10 \mathrm{~m}$, na linha de tráfego, nas profundidades de $0,0-0,2 \mathrm{~m}$ e $0,2-0,4 \mathrm{~m}$. Os pontos da malha de dimensão $100 \times 100 \mathrm{~m}$, totalizando 100 pontos, em uma área de 1 ha, foram georreferenciados.

Foram abertas 100 trincheiras de $0,60 \mathrm{~m}$ de profundidade $(0,3 \times 0,3 \mathrm{~m}$ de largura), para determinação dos atributos DMG, agregados na classe $>2 \mathrm{~mm}$, agregados

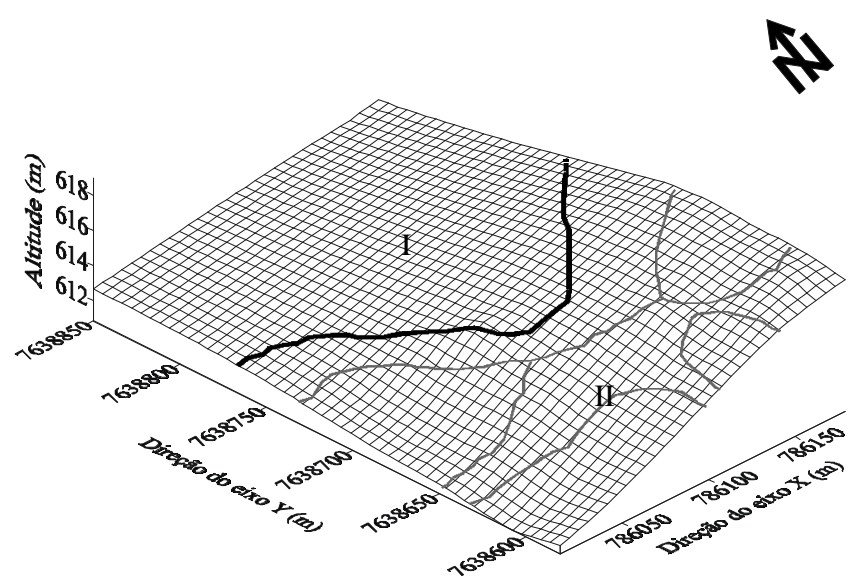

Figura 1. Modelo de elevação digital da área de estudo, com compartimentos identificados (I e II). 
na classe 2-1 mm e MO. As amostras foram levemente destorroadas, de forma manual, e passadas em peneira de 9,51 mm de diâmetro de malha, secadas à sombra, para as análises relativas à agregação, e parte das amostras foi passada na peneira de $2,0 \mathrm{~mm}$, para determinação da matéria orgânica. A separação e estabilidade dos agregados foram determinadas segundo Kemper \& Chepil (1965), com modificações nas seguintes classes de diâmetro: $>2,0 ; 2,0-1,0 ; 1,0-0,25$; $0,25-0,10 ; 0,10-0,05$ e $<0,05 \mathrm{~mm}$. Os agregados foram colocados em contato com a água sobre a peneira de $4,76 \mathrm{~mm}$ por quinze minutos; a massa do material retido em cada peneira foi colocada em estufa a $105^{\circ} \mathrm{C}$. Os resultados foram expressos em porcentual dos agregados retidos nas peneiras $>2 \mathrm{~mm}, 2-1 \mathrm{~mm}$ e diâmetro médio geométrico (DMG). A determinação da matéria orgânica foi realizada conforme Raij et al. (1987).

A variabilidade do solo foi, primeiramente, avaliada pela análise exploratória dos dados, calculando-se a média, mediana, variância, coeficiente de variação, coeficiente de assimetria e coeficiente de curtose, máximo e mínimo. A hipótese de normalidade dos dados foi testada pelo teste de Kolmogorov-Smirnov (SAS Institute, 1995). A observação de valores extremos (máximos e mínimos) e distribuição de frequiência revelaram a existência de valores atípicos em relação às variáveis em estudo. Optou-se pela reedição dos dados extremos, pela média de seus vizinhos.

A teoria das variáveis regionalizadas dispõe de diferentes métodos de análise da variação espacial; um deles, o semivariograma (Vieira et al., 1983; Robertson, 1998), pressupõe a estacionaridade da hipótese intrínseca e pode ser definido como:

$\gamma(\mathrm{h})=\frac{1}{2} \mathrm{E}\left[\mathrm{Z}\left(\mathrm{x}_{\mathrm{i}}\right)-\mathrm{Z}\left(\mathrm{x}_{\mathrm{i}}+\mathrm{h}\right)\right]^{2}$,

a qual é uma função do vetor $h$ e, portanto, depende da magnitude e da direção $h$.

A semivariância é estimada pela expressão:

$\hat{\gamma}(h)=\frac{1}{2 N(h)} \sum_{i=1}^{N(h)}\left[Z\left(x_{i}\right)-Z\left(x_{i}+h\right)\right]^{2}$

em que $\mathrm{N}(\mathrm{h})$ é o número de pares de pontos medidos $\mathrm{Z}\left(\mathrm{x}_{\mathrm{i}}\right), \mathrm{Z}\left(\mathrm{x}_{\mathrm{i}}+\mathrm{h}\right)$, separados por um vetor $\mathrm{h}$. $\mathrm{O}$ gráfico de $\hat{\gamma}(\mathrm{h})$ contra os valores correspondentes de h é denominado semivariograma.

Do ajuste de um modelo matemático aos valores calculados de $\hat{\gamma}(\mathrm{h})$ são definidos os coeficientes do modelo teórico para o semivariograma (o efeito pepita, $\mathrm{C}_{0}$; variância estrutural, $\mathrm{C}_{1}$; patamar, $\mathrm{C}_{0}+\mathrm{C}_{1}$; e o alcance, a).
O efeito pepita é o valor da semivariância para distância zero e representa o componente da variação ao acaso; o patamar é o valor da semivariância em que a curva estabiliza sobre um valor constante; o alcance é a distância da origem até onde o patamar atinge valores estáveis, expressando a distância além da qual as amostras não são correlacionadas (Vieira et al., 1983; Trangmar et al., 1985). Foram ajustados aos dados os seguintes modelos: (a) esférico (Esf), $\hat{\gamma}(\mathrm{h})=\mathrm{C}_{0}+\mathrm{C}_{1}[1,5$ (h/a) $\left.-0,5(\mathrm{~h} / \mathrm{a})^{3}\right]$ para $0<\mathrm{h}<\mathrm{a}$ e $\hat{\gamma}(\mathrm{h})=\mathrm{C}_{0}+\mathrm{C}_{1}$ para $\mathrm{h}>\mathrm{a}$; (b) exponencial (Exp), $\hat{\gamma}(\mathrm{h})=\mathrm{C}_{0}+\mathrm{C}_{1}[1-\exp (-3 \mathrm{~h} / \mathrm{a})]$ para $0<\mathrm{h}<\mathrm{d}$, onde $\mathrm{d}$ é a distância máxima na qual o semivariograma é definido.

Na determinação da existência ou não da dependência espacial, utilizou-se o exame de semivariogramas, por meio do programa $\mathrm{GS}^{+}$(Robertson, 1998). Em caso de dúvida entre mais de um modelo para o mesmo semivariograma, considerou-se o melhor $\mathrm{R}^{2}$ (coeficiente de determinação), menor SQR (soma de quadrados do resíduo) e validação dos modelos por meio da técnica de "Jack Knifing". Na elaboração dos mapas de distribuição espacial das variáveis foi utilizado o programa Surfer (Golden Software Inc., 1999).

\section{Resultados e Discussão}

Os resultados referentes à análise descritiva para as variáveis DMG, agregados na classe $>2 \mathrm{~mm}$, agregados na classe 2-1 mm e MO indicaram ajuste à distribuição normal (Tabela 1). Os valores da média e mediana, para todas as variáveis, estão próximos, mostrando distribuições simétricas, o que pode ser confirmado pelos valores de assimetria próximos de zero. Os resultados referentes ao teste Kolmogorov-Smirnov indicaram normalidade para DMG em todas as profundidades. Salviano et al. (1998), Araujo (2002) e Carvalho et al. (2003) também não encontraram distribuição normal para a matéria orgânica. Souza et al. (2003) obtiveram resultado oposto a este trabalho, encontrando distribuição normal para a matéria orgânica.

Segundo Isaaks \& Srivastava (1989), mais importante que a normalidade dos dados é a ocorrência ou não do chamado efeito proporcional, que a média e a variabilidade dos dados sejam constantes na área de estudo, 
o que foi observado, ou seja, ocorrência de estacionaridade necessária ao uso da geoestatística. No entanto, como os dados são obtidos na natureza, o ajuste de uma distribuição teórica é apenas aproximado (Warrick \& Nielsen, 1980; Goovaerts, 1997; Webster, 2000).

A matéria orgânica na profundidade de $0,0-0,2 \mathrm{~m}$ apresentou o menor coeficiente de variação $(10,4 \%)$ e o DMG, na profundidade de $0,2-0,4 \mathrm{~m}$, apresentou o maior coeficiente de variação $(34,5 \%)$ (Tabela 1$)$. Adotando o critério de classificação para $\mathrm{CV}$ proposto por Warrick \& Nielsen (1980) para medida estatística de dispersão, seus valores revelaram-se altos, médios e baixos. Foram altos (>24\%), em relação à variável DMG nas profundidades $0,0-0,2 \mathrm{~m}$ e $0,2-0,4 \mathrm{~m}$; Sá et al. (2000), estudando a estabilidade de agregados por meio da estatística clássica, encontraram CV médio para o DMG. Para as variáveis agregados na classe $>2 \mathrm{~mm}$ e agregados na classe $2-1 \mathrm{~mm}$, nas profundidades de $0,0-0,2 \mathrm{~m}$ e $0,2-0,4 \mathrm{~m}$, e para a variável MO na profundidade de 0,2-0,4 $\mathrm{m}$ foram encontrados valores de $\mathrm{CV}$ médios, concordando com os resultados obtidos por Souza et al. (1997, 2003). Na variável MO, na profundi- dade de $0,0-0,2 \mathrm{~m}$, os valores de $\mathrm{CV}$ foram baixos (12\%), sendo que este resultado está de acordo com o de Pocay (2000) e Araujo (2002).

Muitos autores têm adotado essas classes de CV em trabalhos relacionados com a variabilidade espacial do solo (Macedo et al., 1998; Souza et al., 2001). Apesar de os coeficientes de variação permitirem comparar a variabilidade entre amostras com unidades diferentes, $\mathrm{o}$ seu emprego não deve ser generalizado, devendo-se apreciar esses resultados segundo as finalidades a que o trabalho se destina. $\mathrm{O}$ coeficiente de variação foi menor na camada de $0,0-0,2 \mathrm{~m}$ em relação à camada de $0,2-0,4 \mathrm{~m}$ para todas as variáveis (Tabela 1 ).

Os resultados da análise geoestatística mostraram que todas as variáveis analisadas apresentaram dependência espacial nas profundidades em estudo (Tabela $2 \mathrm{e}$ Figura 2). O modelo esférico ajustou-se para todas as variáveis nas profundidades de $0,0-0,2 \mathrm{~m}$ e $0,2-0,4 \mathrm{~m}$, com exceção para agregados na classe $2-1 \mathrm{~mm}$ na profundidade de 0,2-0,4 $\mathrm{m}$ que se ajustou ao modelo exponencial. Com relação ao ajuste da variável matéria orgânica, os resultados concordam com os obtidos por Souza et al. (1998) e Silva \& Chaves (2001), nas profundidades de $0,0-0,2 \mathrm{~m}$ e $0,2-0,4 \mathrm{~m}$.

Tabela 1. Estatística descritiva em relação ao diâmetro médio geométrico (DMG) e porcentagem dos agregados na classe $>2$ mm e na classe 2-1 mm e em relação à matéria orgânica (MO) de 100 amostras coletadas na malha nas profundidades de $0,0-0,2 \mathrm{~m}$ e $0,2-0,4 \mathrm{~m}$.

\begin{tabular}{|c|c|c|c|c|}
\hline Estatística & DMG $(\mathrm{mm})$ & $>2 \mathrm{~mm}(\%)$ & $2-1 \mathrm{~mm}(\%)$ & $\left(\mathrm{MO} \mathrm{g} \mathrm{dm}^{-3}\right)$ \\
\hline \multicolumn{5}{|c|}{$0,0-0,2 \mathrm{~m}$} \\
\hline Média & 2,24 & 60,21 & 55,94 & 27,01 \\
\hline Mediana & 2,30 & 59,22 & 56,97 & 27,50 \\
\hline Mínimo & 0,63 & 50,18 & 46,58 & 21,01 \\
\hline Máximo & 4,04 & 71,92 & 66,36 & 33,01 \\
\hline Variância & 0,56 & 42,12 & 20,98 & 8,25 \\
\hline Assimetria & $-0,09$ & 0,20 & $-0,29$ & $-0,27$ \\
\hline Curtose & $-0,68$ & $-1,34$ & $-0,43$ & $-0,19$ \\
\hline CV $(\%)$ & 33,40 & 20,45 & 20,05 & 10,37 \\
\hline $\mathrm{p}^{(1)}$ & $0,07^{*}$ & 0,11 & 0,14 & 0,13 \\
\hline \multicolumn{5}{|c|}{$0,2-0,4 \mathrm{~m}$} \\
\hline Média & 2,28 & 19,98 & 22,59 & 23,07 \\
\hline Mediana & 2,30 & 20,45 & 22,69 & 23,00 \\
\hline Mínimo & 0,46 & 11,72 & 16,76 & 15,00 \\
\hline Máximo & 4,27 & 28,15 & 29,65 & 30,00 \\
\hline Variância & 0,58 & 15,11 & 8,23 & 14,50 \\
\hline Assimetria & 0,01 & $-0,21$ & 0,32 & $-0,12$ \\
\hline Curtose & $-0,16$ & $-0,71$ & $-0,17$ & $-0,86$ \\
\hline CV (\%) & 34,45 & 23,44 & 21,45 & 16,02 \\
\hline $\mathrm{p}^{(1)}$ & $0,05^{*}$ & 0,12 & 0,11 & 0,13 \\
\hline
\end{tabular}

(1) Estatística do teste de Kolmogorov-Smirnov. *Significativo a $5 \%$ de probabilidade. 
O efeito pepita é um parâmetro importante do semivariograma e indica variabilidade não explicada, considerando a distância de amostragem utilizada (McBratney \& Webster, 1986). Esse parâmetro pode ser expresso como porcentagem do patamar, com o objetivo de facilitar a comparação do grau de dependência espacial das variáveis em estudo (Trangmar et al., 1985). Na análise do grau de dependência espacial das variáveis em estudo, utilizou-se a classificação de Robertson et al. (1998). A análise da relação $C_{1} /\left(C_{0}+C_{1}\right)$ das variáveis $\mathrm{DMG}$, agregados na classe $>2 \mathrm{~mm}$, agregados na classe $2-1 \mathrm{~mm}$ e MO mostrou que as variáveis apresentaram dependência espacial moderada na profundidade de $0,0-0,2 \mathrm{~m}$. Na profundidade de $0,2-0,4 \mathrm{~m}$, as variáveis apresentaram dependência espacial forte, com exceção da variável agregados na classe $2-1 \mathrm{~mm}$ que apresentou dependência espacial moderada (Tabela 2). O comportamento espacial da matéria orgânica concorda com os resultados obtidos por Souza et al. (1998, 2003) e Araujo (2002).

Os valores dos alcances relativos aos semivariogramas têm uma importância considerável na determinação do limite da dependência espacial, o que pode ser também um indicativo do intervalo entre unidades de mapeamento de solos (Trangamar et al., 1985;
Webster, 2000). As variáveis apresentaram diferentes alcances de dependência espacial, nas profundidades de $0,0-0,2 \mathrm{~m}$ e $0,2-0,4 \mathrm{~m}$. A variável $\mathrm{MO}$ apresentou o maior alcance $(77 \mathrm{~m})$ na profundidade de $0,0-0,2 \mathrm{~m}$, e os agregados na classe $>2 \mathrm{~mm}$ apresentaram o menor alcance $(21 \mathrm{~m})$ na profundidade de $0,2-0,4 \mathrm{~m}$. Na profundidade de $0,2-0,4 \mathrm{~m}$, os alcances foram os menores, e houve uma diminuição do alcance em profundidade em todas as variáveis, indicando a maior continuidade na distribuição espacial das propriedades do solo na camada mais superficial. Esses resultados corroboram os de Souza et al. (2001).

Os mapas das variáveis DMG, agregados na classe $>2 \mathrm{~mm}$, agregados na classe $2-1 \mathrm{~mm}$ e MO na profundidade de 0,0-0,2 $\mathrm{m}$, apresentaram valores entre 1,3 e $2,7 \mathrm{~mm}, 54$ e $66 \%, 17$ e $23 \%$ e 25 e $30 \mathrm{~g} \mathrm{dm}^{-3}$, respectivamente (Figura 3 ). Na profundidade de $0,2-0,4 \mathrm{~m}$, os valores variaram entre 1,3 e 2,9 mm, $49 \mathrm{e}$ $65 \%, 20$ e $26 \%$ e 19 e $26 \mathrm{~g} \mathrm{dm}^{-3}$, respectivamente. Houve correlação espacial do DMG e classes de agregados com o teor de matéria orgânica (Figura 3). Ocorreu redução dos valores da variável agregados na classe $>2 \mathrm{~mm}$ e um aumento de valores da variável agregados na classe $2-1 \mathrm{~mm}$ na profundidade de $0,2-0,4 \mathrm{~m}$, refletindo a influência do manejo da cultura da cana-de-açú-

Tabela 2. Modelos e parâmetros estimados dos semivariogramas experimentais em relação ao diâmetro médio geométrico (DMG, mm) e porcentagem dos agregados na classe $>2 \mathrm{~mm}$, e na classe $2-1 \mathrm{~mm}(\%)$ e em relação à matéria orgânica

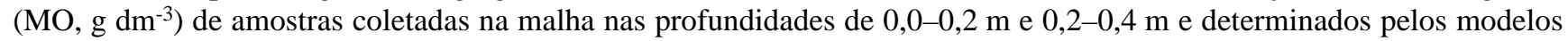
esférico e exponencial.

\begin{tabular}{|c|c|c|c|c|}
\hline Parâmetro & DMG & $>2 \mathrm{~mm}$ & $2-1 \mathrm{~mm}$ & $\mathrm{MO}$ \\
\hline \multicolumn{5}{|c|}{$0,0-0,2 \mathrm{~m}$} \\
\hline Modelo & Esférico & Esférico & Esférico & Esférico \\
\hline Efeito pepita $\left(\mathrm{C}_{0}\right)$ & 0,05 & 24,31 & 6,27 & 1,45 \\
\hline Patamar $\left(\mathrm{C}_{1}\right)$ & 0,14 & 24,32 & 6,60 & 1,55 \\
\hline Alcance (a) & 34 & 70 & 68 & 77 \\
\hline$\left[\mathrm{C}_{1} /\left(\mathrm{C}_{0}+\mathrm{C}_{1}\right)\right] \times 100^{(1)}$ & 74 & 50 & 51 & 51 \\
\hline $\mathrm{R}^{2}(\%)^{(2)}$ & 98 & 90 & 95 & 98 \\
\hline $\mathrm{SQR}^{(3)}$ & 1,69 E-04 & 50,91 & 1,82 & 0,04 \\
\hline \multicolumn{5}{|c|}{$0,2-0,4 \mathrm{~m}$} \\
\hline Modelo & Esférico & Esférico & Exponencial & Esférico \\
\hline Efeito pepita $\left(\mathrm{C}_{0}\right)$ & 0,04 & 0,71 & 4,41 & 0,21 \\
\hline Patamar $\left(\mathrm{C}_{1}\right)$ & 0,15 & 9,42 & 4,52 & 5,94 \\
\hline Alcance (a) & 31 & 21 & 51 & 60 \\
\hline$\left[\mathrm{C}_{1} /\left(\mathrm{C}_{0}+\mathrm{C}_{1}\right)\right] \times 100$ & 79 & 93 & 50 & 96 \\
\hline $\mathrm{R}^{2}(\%)$ & 95 & 94 & 90 & 98 \\
\hline SQR & 4,67 E-04 & 0,84 & 1,06 & 0,45 \\
\hline
\end{tabular}

${ }^{(1)}\left[C_{1} /\left(C_{0}+C_{1}\right)\right] x 100$ : grau de dependência espacial. ${ }^{(2)}$ Coeficiente de determinação. ${ }^{(3)}$ Soma dos quadrados dos resíduos. 
car, que recebe um tráfego de máquinas pesadas excessivo e queima dos resíduos. Corrêa (2002), comparando sistemas de preparo com mata nativa, verificou que a agregação na classe $>2 \mathrm{~mm}$ diminui de $88,1 \%$ para 63,9\% em um Latossolo Vermelho-Amarelo.

$\mathrm{Na}$ área onde o relevo possui forma linear (Compartimento I), observou-se os menores valores para os atributos DMG, agregados na classe $>2 \mathrm{~mm}$ e maiores valores de agregados na classe $2-1 \mathrm{~mm}$, coincidindo com os menores valores de $\mathrm{MO}$ em ambas profundidades. Nesse compartimento, todas as variáveis apresentaram
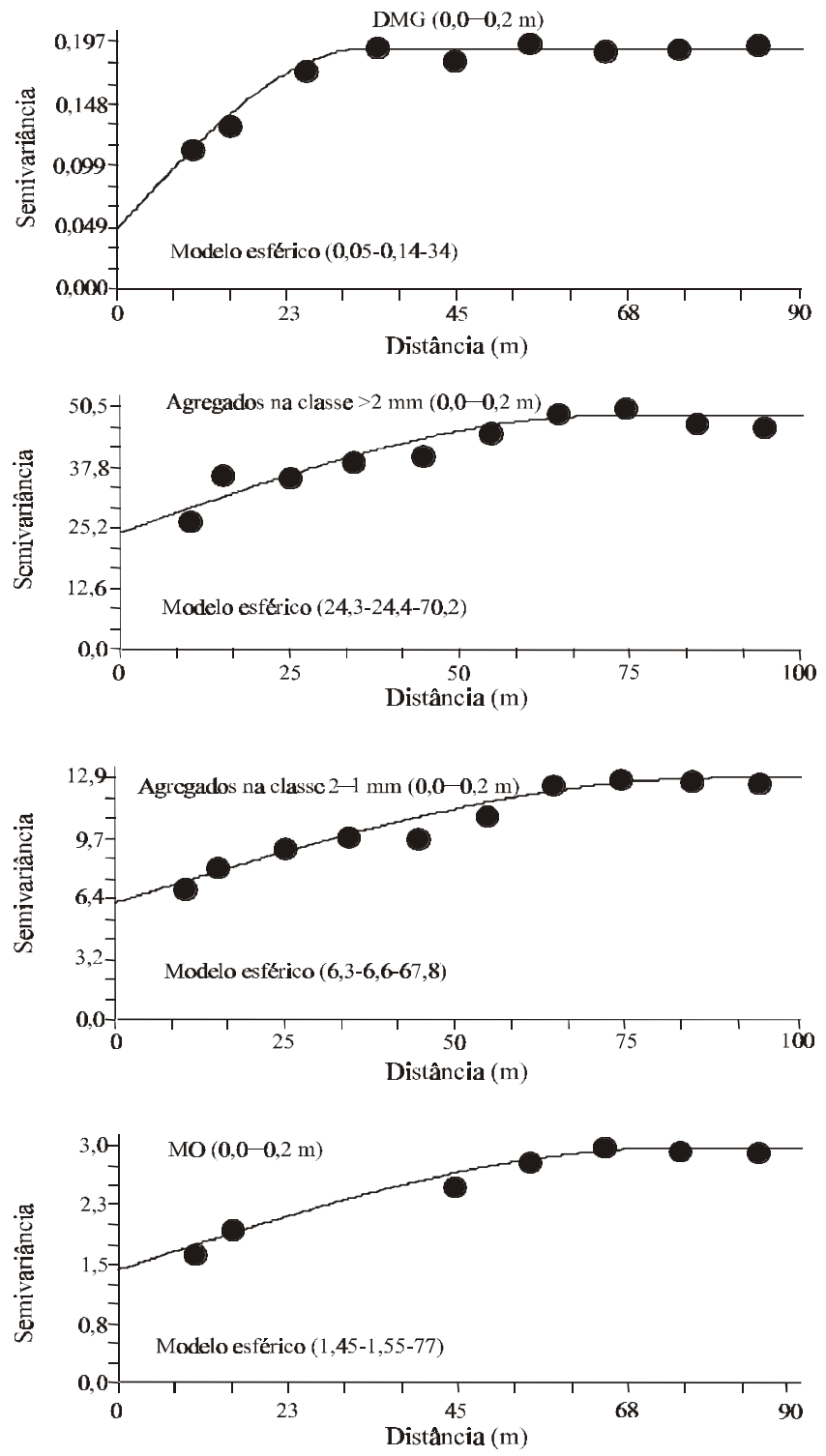

menor variabilidade; já na área com curvaturas côncavas e convexas (compartimento II), a variação é relativamente maior (Figuras 2 e 3). Shinjo et al. (2000) encontraram relação entre posição da paisagem e variabilidade espacial de estabilidade de agregados, informando que, em áreas mais planas, a estabilidade de agregados apresentou um comportamento mais definido que em áreas mais declivosas. Bird et al. (2002) e Le Bissonnais et al. (2002) encontraram relação da estabilidade de agregados com diferentes posições na paisagem.
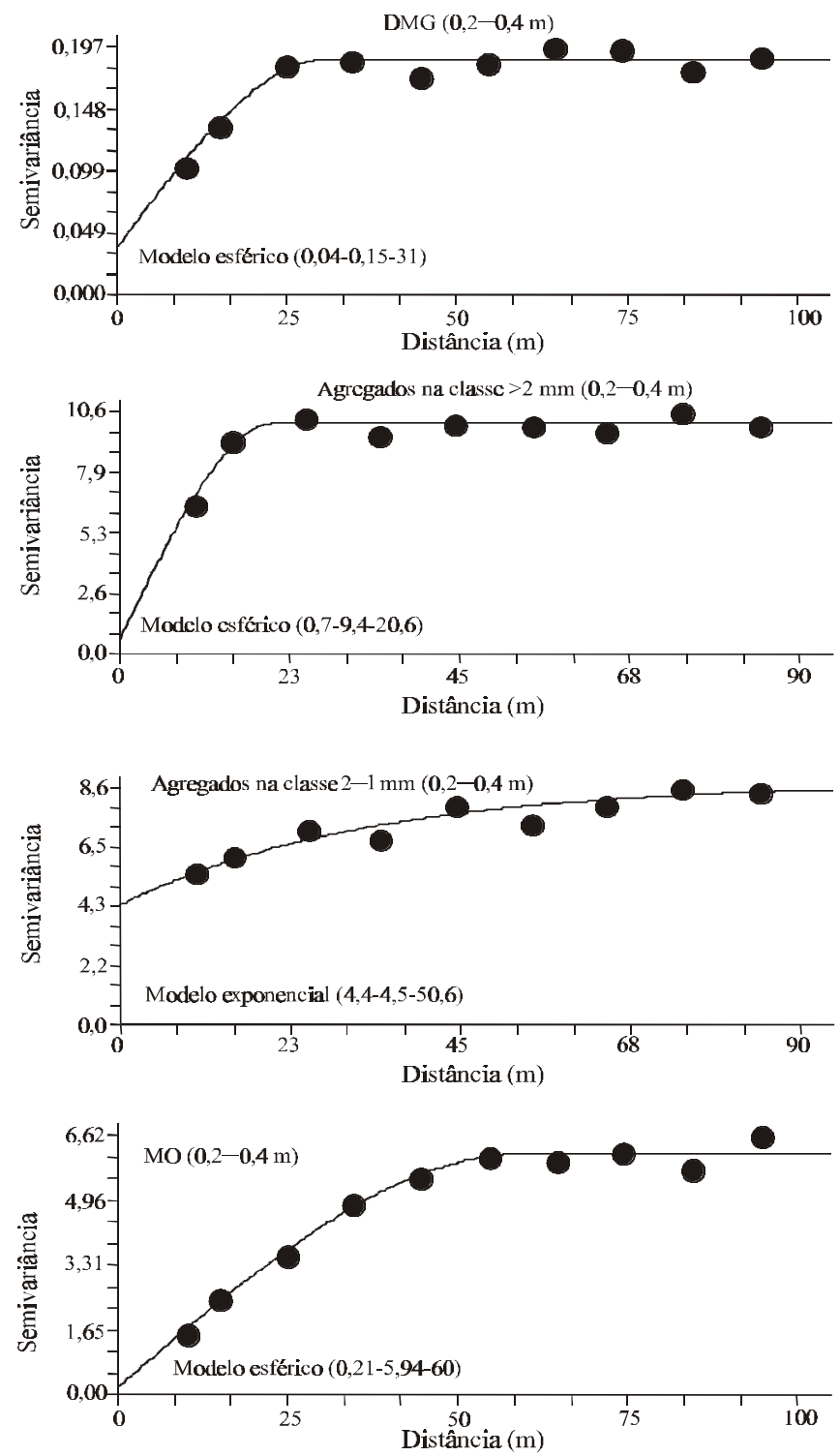

Figura 2. Semivariogramas das variáveis diâmetro médio geométrico (DMG, mm), agregados na classe >2 mm (\%), agregados na classe 2-1 mm (\%) e $\mathrm{MO}\left(\mathrm{g} \mathrm{dm}^{-3}\right)$ nas profundidades de 0,0-0,2 m e 0,2-0,4 m, determinados pelos modelos ajustados esférico e exponencial $\left(\mathrm{C}_{0}\right.$ : efeito pepita; $\mathrm{C}=\mathrm{C}_{0}+\mathrm{C}_{1}$ : patamar; a: alcance $)$. 

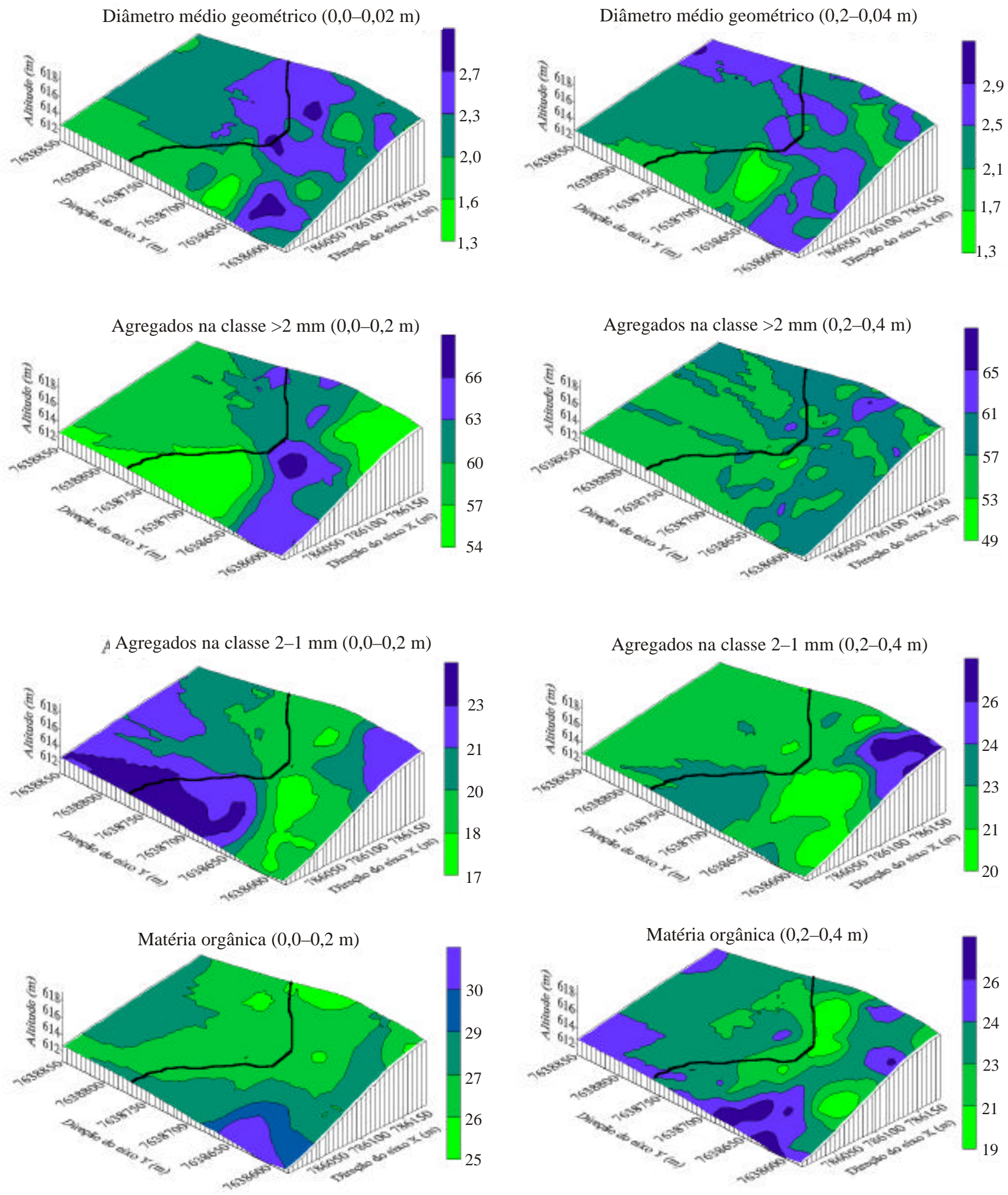

Figura 3. Distribuição espacial das variáveis diâmetro médio geométrico (DMG, mm), agregados na classe >2 mm (\%), agregados na classe 2-1 mm (\%) e matéria orgânica $\left(\mathrm{MO}, \mathrm{g} \mathrm{dm}^{-3}\right)$ nas profundidades de $0,0-0,2 \mathrm{~m}$ e 0,2-0,4 m. 


\section{Conclusões}

1. As variáveis estudadas apresentam estrutura de dependência espacial, e os maiores alcances são observados na profundidade de $0,0-0,2 \mathrm{~m}$.

2. A matéria orgânica apresenta comportamento espacial similar com a estabilidade de agregados.

3. Pequenas variações no gradiente do declive e nas formas do relevo condicionam variabilidade espacial diferenciada em relação à matéria orgânica e estabilidade de agregados nas profundidades estudadas.

\section{Agradecimentos}

À Fundação de Amparo à Pesquisa do Estado de São Paulo, pela concessão de bolsa ao primeiro autor e auxílio financeiro para o desenvolvimento do projeto; à Usina São Martinho, pela concessão da área para o experimento.

\section{Referências}

ARAUJO, A.A.V. Variabilidade espacial de propriedades químicas e granulométricas do solo na definição de zonas homogêneas de manejo. 2002. 80p. Dissertação (Mestrado) Universidade do Estado de São Paulo, Jaboticabal.

BIRD, S.B.; HERRICK, J.E.; WANDER, M.M.; WRIGHT, S.F. Spatial heterogeneity of aggregate stability and soil carbon in semiarid rangeland. Environmental Pollution, v.116, p.445-455, 2002. BURROUGH, P.A.; BOUMA, J.; YATES, S.R. The state of the art in pedometrics. Geoderma, v.62, p.311-326, 1994.

CARVALHO, M.P.; TAKEDA, E.Y.; FREDDI, O.S. Variabilidade espacial de atributos de um solo sob videira em Vitória Brasil (SP). Revista Brasileira de Ciência do Solo, v.27, p.695-703, 2003.

CORRÊA, J.C. Efeito de sistemas de cultivo na estabilidade de agregados de um Latossolo Vermelho-Amarelo em Querência, MT. Pesquisa Agropecuária Brasileira, v.37, p.203-209, 2002.

EMBRAPA. Centro Nacional de Pesquisa de Solos (Rio de Janeiro, RJ). Sistema brasileiro de classificação de solos. Brasília: Embrapa-SPI; Embrapa-CNPS, 1999. 412p.

FRAISSE, C.W.; SUDDUTH, K.A.; KITCHEN, N.R.; FRIDGEN, J.J. Use of unsupervised clustering algorithms of delineating within-field management zones. St. Joseph: American Society of Agricultural Engineers, 1999. 121p. (ASAE Paper, 15).

GOLDEN SOFTWARE INC. (Golden, Estados Unidos). SURFER for Windows: realese 7.0: contouring and 3D surface mapping for scientist's engineers, user's guide. New York, 1999. 619p.

GOOVAERTS, P. Geoestatistics for natural resources evaluation. New York: Oxford University Press, 1997. 483p.

ISAAKS, E.H.; SRIVASTAVA, R.M. An introduction to applied geoestatistics. New York: Oxford University Press, 1989. 561p.
KEMPER, W.D.; CHEPIL, W.S. Size distribution of aggregates. In: BLACK, C.A. (Ed.). Methods of soil analysis. Madison: American Society of Agronomy, 1965. p.499-510.

LE BISSONNAIS, Y.; CROSS-CAYOT, S.; GASCUEL-ODOUX, C. Topographic dependence of aggregate stability, overland flow and sediment transport. Agronomie, v.22, p.489-501, 2002.

McBRATNEY, A.B.; WEBSTER, R. Choosing functions for semivariograms of soil properties and fitting them to sampling estimates. Journal of Soil Science, v.37, p.617-639, 1986.

MACEDO, J.R.; OTONNI FILHO, T.B.; MENEGUELLI, N.A. Variabilidade de características físicas, químicas e físico-hídricas em solo Podzólico Vermelho-Amarelo de Seropédica, RJ. Pesquisa Agropecuária Brasileira, v.33, p.2043-2053, 1998.

MARQUES JÚNIOR, J.; LEPSCH, I.F. Depósitos superficiais neocenozóicos, superfícies geomórficas e solos em Monte Alto, SP. Geociências, v.19, p.265-281, 2000.

MULLA, D.J.; BATÍ, A.U.; HAMMOND, M.W.; BENSON, J.A. A comparison of winter wheat yield and quality under uniform versus spatially variable fertilizer management. Agriculture Environment, v.38, p.301-311, 1992.

PERIN, A.; GUERRA, J.G.M.; TEIXEIRA, M.G.; PEREIRA, M.G.; FONTANA, A. Efeito da cobertura viva com leguminosas herbáceas perenes na agregação de um argissolo. Revista Brasileira de Ciência do Solo, v.26, p.713-720, 2002.

PIERCE, F.J.; NOWAK, P. Aspects of precision agriculture. Advances in Agronomy, v.67, p.1-85, 1999.

POCAY, V.G. Relações entre pedoforma e variabilidade espacial de atributos de latossolos sob cultivo intensivo de cana-de-açúcar. 2000. 177p. Dissertação (Mestrado) - Universidade do Estado de São Paulo, Jaboticabal.

RAIJ, B. van; QUAGGIO, J.A.; CANTARELLA, J.; FERREIRA, M.E.; LOPES, A.S.; BATAGLIA, O. Análise química do solo para fins de fertilidade. Campinas: Fundação Cargill, 1987. 170p. ROBERTSON, G.P. GS $^{+}$geostatistics for the environmental sciences: GS ${ }^{+}$user's guide. Plainwell: Gamma Design Software, 1998. $152 \mathrm{p}$.

SÁ, M.A.C.; LIMA, J.M.; SILVA, M.L.N.; DIAS JUNIOR, M.S. Comparação entre métodos para estudo da estabilidade de agregados em solos. Pesquisa Agropecuária Brasileira, v.35, p.1825-1834, 2000.

SALVIANO, A.A.C.; VIEIRA, S.R.; SPAROVEK, G. Variabilidade espacial de atributos de solo e de Crotalaria juncea (L.) em área severamente erodida. Revista Brasileira de Ciência do Solo, v.22, p.115-122, 1998.

SAS INSTITUTE (Cary, Estados Unidos). Statistical analysis system for Windows: computer program manual. Cary, 1995. 705p.

SHINJO, H.; FUJITA, H.; GINTZBURGER, G.; KOSAKI, T. Soil aggregate stability under different landscapes and vegetation types in semiarid area in northeastern Syria. Soil Science and Plant Nutrient, v.46, p.229-240, 2000. 
SILVA, I.F.; MIELNICZUK, J. Ação do sistema radicular de plantas na formação e estabilização de agregados do solo. Revista Brasileira de Ciência do Solo, v.21, p.113-117, 1997.

SILVA, P.C.M.; CHAVES, L.H.G. Avaliação e variabilidade espacial de fósforo, potássio e matéria orgânica em alissolos. Revista Brasileira de Engenharia Agrícola e Ambiental, v.5, p.431-436, 2001.

SOUZA, C.K.; MARQUES JÚNIOR, J.; MARTINS FILHO, M.V.; PEREIRA, G.T. Influência do relevo na variação anisotrópica dos atributos químicos e granulométricos de um latossolo em Jaboticabal, SP. Engenharia Agrícola, v.23, p.486-495, 2003.

SOUZA, L.S.; COGO, N.P.; VIEIRA, S.R. Variabilidade de fósforo, potássio e matéria orgânica no solo em relação a sistemas de manejo. Revista Brasileira de Ciência do Solo, v.22, p.77-86, 1998.

SOUZA, L.S.; COGO, N.P.; VIEIRA, S.R. Variabilidade de propriedades físicas e químicas do solo em um pomar cítrico. Revista Brasileira de Ciência do Solo, v.21, p.367-372, 1997.
SOUZA, Z.M.; SILVA, M.L.S.; GUIMARÃES, G.L.; CAMPOS, D.T.S.; CARVALHO, M.P.; PEREIRA, G.T. Variabilidade espacial de atributos físicos em um Latossolo Vermelho distrófico sob semeadura direta em Selvíria (MS). Revista Brasileira de Ciência do Solo, v.25, p.699-707, 2001.

TRANGMAR, B.B.; YOST, R.S.; WADE, M.K.; UEHARA, G. Applications of geostatistics to spatial studies of soil properties. Advances in Agronomy, v.38, p.45-94, 1985.

TROEH, F.R. Landform equations fitted to contour maps. American Journal of Science, v.263, p.616-627, 1965.

VIEIRA, S.R.; HATFIELD, J.L.; NIELSEN, D.R.; BIGGAR, J.W. Geoestatiscal theory and application to variability of some agronomical properties. Hilgardia, v.51, p.1-75, 1983.

WARRICK, A.W.; NIELSEN, D.R. Spatial variability of soil physical properties in the field. In: HILLEL, D. (Ed.). Applications of soil physics. New York: Academic, 1980. p.319-344.

WEBSTER, R. Is soil variation random? Geoderma, v.97, p.149163,2000 . 